\title{
GERMINACIÓN Y VIGOR DE SEMILLAS DE POBLACIONES DE MAÍZ CON DIFERENTE PROPORCIÓN DE ENDOSPERMO VÍTREO
}

\author{
SEED GERMINATION AND VIGOR OF LANDRACE MAIZE \\ POPULATIONS WITH DIFFERENT PROPORTION OF VITREOUS \\ ENDOSPERM
}

\author{
Pedro Guillen-de la Cruz ${ }^{1}$, Ramiro Velázquez-Morales², Efraín de la Cruz-Lázaro²*, César Márquez- \\ Quiroz $^{2}$, Rodolfo Osorio-Osorio ${ }^{2}$ \\ ${ }^{1}$ Instituto Tecnológico Superior de la Región Sierra, Carretera Teapa-Tacotalpa Km 4.5, Francisco Javier \\ Mina, CP. 86801, Tabasco, México. \\ ${ }^{2}$ Universidad Juárez Autónoma de Tabasco, División Académica de Ciencias Agropecuarias, Carretera \\ Villahermosa-Teapa, Km 25, CP. 86298. Centro, Tabasco, México. \\ * Autor para correspondencia E-mail: efrain.delacruz@ujat.mx
}

\section{RESUMEN}

Los atributos de calidad fisiológica de semillas son componentes importantes para ampliar la caracterización del germoplasma. Se evaluaron 35 poblaciones de maíz (Zea mays L.) criollo con diferente tipo de endospermo, con el objetivo de establecer la relación entre el endospermo vítreo con la germinación de semillas y vigor de plántulas. El estudio se realizó en dos fases: en la primera fase se determinó la textura por disección, para determinar el peso de endospermo harinoso, endospermo vítreo, pericarpio, germen y peso de grano; $y$ en la segunda fase se realizaron pruebas fisiológicas de germinación (\%), vigor (longitud de plántula), número de plantas normales, número de plantas anormales y número de semillas muertas. Se encontraron diferencias $(p \leq 0,01)$ entre las poblaciones en las características estructurales y fisiológicas del grano. El análisis de correlación canónica detectó que en la variable canónica estructural 1, las variables con mayor influencia fueron peso de endospermo vítreo y peso de grano. Mientras que la variable fisiológica 1 estuvo asociada con las variables plantas anormales, semillas muertas, vigor y germinación. De acuerdo al valor de $r=$ 0.7051, las características estructurales están directamente relacionadas con la fisiología de la semilla.

Palabras clave: Zea mays L., endospermo vítreo, endospermo harinoso, germinación.

\section{ABSTRACT}

The physiological seed quality attributes assayed in the laboratory are important components to extend the characterization of germplasm. Thirty-five landrace maize (Zea mays L.) populations with different types of endosperm were evaluated to determine the relationship between vitreous endosperm, seed germination and seedling vigor. The study was carried into two phases. During the first phase, the following structural characteristics were determined: grain weight, floury endosperm weight, vitreous endosperm weight, pericarp weight, germ weight and grain weight; while, in the second phase, germination rate (\%), vigor (seedling length), number of normal plants, number of abnormal plants and number of dead seeds were determined. Differences $(p \leq 0.01)$ in the structural and physiological characteristics of the seeds were found between landrace maize populations. The comparison of means by Tukey test $(\mathrm{p} \leq 0.05)$ detected differences between maize populations in 
terms of structural and physiological variables. The canonical correlation analysis revealed that the most influential variables in the structural canonical variable 1 were vitreous endosperm weight and grain weight, while the physiological variable 1 was associated with abnormal plants, dead seeds, vigor and germination. According to the value $r=0.7051$, structural characteristics are directly related to the physiology of the seed.

Key words: Zea mays L., vitreous endosperm, floury endosperm, germination, corn.

\section{INTRODUCCIÓN}

México es considerado el centro de origen, domesticación y dispersión del maíz (Zea mays L.), con 59 razas potencialmente diferentes, que agrupan a cientos de maíces criollos (Kato et al., 2009). Estos tienen importancia social y económica, por lo que es necesario llevar a cabo trabajos y programas de investigación en producción de semillas que permitan evaluar e identificar la calidad en germinación y vigor, para garantizar el rendimiento y productividad (Vitoria y Méndez, 2007). El concepto de calidad se basa en la valoración de atributos que mejoran el establecimiento de la planta en el campo, destacando los genéticos, fisiológicos, físicos y sanitarios (Pérez et al., 2006). La calidad de la semilla implica la integridad morfológica y los procesos fisiológicos que conservan la viabilidad, siendo indicadores de la calidad la germinación y el vigor, los cuales dependen del genotipo y del manejo en la producción y poscosecha (González et al., 2014).

La germinación de la semilla se define como la serie de procesos metabólicos y morfogenéticos, que transforman el embrión en una plántula que se puede convertir en una planta adulta (Rosental et al., 2014). El vigor es la sumatoria de aquellas propiedades de las semillas que determinan el nivel de actividad y la respuesta durante la germinación y emergencia de la plántula (Navarro et al. 2015). Es por ello que las pruebas de germinación y vigor se recomiendan implementarlas de forma paralela, para emitir veredictos integrales de la calidad fisiológica, debido a que estas dos pruebas infieren y ayudan a definir la calidad física y fisiológica de la semilla (Pérez et al., 2006).

En maíz se puede encontrar una gran diversidad en tamaño, forma y composición de la semilla, debido a factores genéticos, ambientales y la ubicación de ésta en la mazorca (Pérez et al., 2007). Las diferencias estructurales de mayor importancia en la semilla son: tamaño, forma y contenido de endospermo vítreo y harinoso, este último constituye alrededor del $80 \%$ de la semilla, seguido del germen con el $12 \%$, pericarpio con $5 \%$, y pedicelo con $1 \%$ (Vázquez et al., 2010). Al respecto Pérez et al. (2007) al evaluar la calidad fisiológica en semillas de maíces con diferencias estructurales, encontraron respuesta diferencial en la calidad fisiológica de los genotipos evaluados. Con respecto a los maíces criollos, se requiere estudiar la relación que existe entre el tipo de endospermo y la calidad física y fisiológica de la semilla. Debido a lo anterior, el presente trabajo, tuvo el objetivo de evaluar la relación entre la proporción de endospermo vítreo con la germinación de semillas y vigor de plántulas de 35 poblaciones de maíces criollos de la región Grijalva del estado de Tabasco, México.

\section{MATERIALES Y MÉTODOS}

El estado de Tabasco se localiza entre $90^{\circ} 58^{\prime} 08^{\prime \prime}$ y $94^{\circ} 07^{\prime} 00^{\prime \prime} \mathrm{LO}$, y entre $17^{\circ} 15^{\prime} 00^{\prime \prime}$ y $18^{\circ} 38^{\prime} 45^{\prime \prime}$ de LN, sobre la llanura costera del Golfo de México. Tiene una extensión territorial de $24738 \mathrm{~km}^{2}$, que representan el 1,3\% del territorio nacional. El clima predominante es cálido húmedo con abundantes lluvias en verano (INEGI, 2011), precipitación promedio entre 2500 y $3000 \mathrm{~mm}$, con temperatura media anual de $25^{\circ} \mathrm{C}$ (Ruíz-Álvarez et al., 2012). Se divide en dos regiones económicas, la región del Grijalva que comprende las subregiones Centro, Chontalpa y Sierra, y la región Usumacinta que comprende las subregiones Ríos y Pantanos.

Se recolectaron 35 poblaciones de maíces criollos, de octubre a diciembre de 2015, en la cosecha de las siembras de año (PrimaveraVerano) de maíz con frijol (Phaseolus vulgaris L.) o maíz con calabaza (Cucurbita spp.) conocida como milpa (Mariaca et al., 2015), en 10 municipios de la región Grijalva del estado de Tabasco, de las cuales nueve se realizaron en la subregión Centro, siete en la subregión Chontalpa y 19 en la subregión Sierra (Tabla 1). Para determinar que fueran maíces criollos se realizaron las recolectas sólo con agricultores que manifestaron estar sembrando su maíz en los últimos 10 años. De cada población se recolectaron al azar en la troje del agricultor entre 20 y 30 mazorcas, las cuales se desgranaron de forma manual, para luego determinar el contenido de humedad y estandarizar a un contenido del $14 \%$ para su almacenamiento a temperatura ambiente en el laboratorio hasta su uso. Del total de granos obtenidos de las mazorcas recolectadas de cada población se tomó la muestra para realizar los análisis de variables estructurales y fisiológicas 
Tabla 1. Número de población, municipio y datos geográficos de 35 poblaciones de maíz criollo colectado en la Región Grijalva del estado de Tabasco, México.

Table 1. Population number, municipality and geographic data of 35 landrace maize populations collected in the Grijalva region of the state of Tabasco, Mexico.

\begin{tabular}{|c|c|c|c|c|}
\hline Población & Municipio & Subregión & Latitud Norte & Longitud Oeste \\
\hline 01 & Centro & Centro & $18^{\circ} 09^{\prime} 42^{\prime \prime}$ & $92^{\circ} 47^{\prime} 02^{\prime \prime}$ \\
\hline 02 & Centro & Centro & $18^{\circ} 09^{\prime} 43^{\prime \prime}$ & $92^{\circ} 47^{\prime} 03^{\prime \prime}$ \\
\hline 03 & Centro & Centro & $18^{\circ} 09^{\prime} 41^{\prime \prime}$ & $92^{\circ} 47^{\prime} 01^{\prime \prime}$ \\
\hline 04 & Tacotalpa & Sierra & $17^{\circ} 24^{\prime} 15^{\prime \prime}$ & $92^{\circ} 41^{\prime} 06^{\prime \prime}$ \\
\hline 05 & Tacotalpa & Sierra & $17^{\circ} 24^{\prime} 15^{\prime \prime}$ & $92^{\circ} 41^{\prime} 07^{\prime \prime}$ \\
\hline 06 & Tacotalpa & Sierra & $17^{\circ} 37^{\prime} 46^{\prime \prime}$ & $92^{\circ} 45^{\prime} 12^{\prime \prime}$ \\
\hline 07 & Tacotalpa & Sierra & $17^{\circ} 25^{\prime} 14^{\prime \prime}$ & $92^{\circ} 47^{\prime} 39^{\prime \prime}$ \\
\hline 08 & Nacajuca & Centro & $18^{\circ} 11^{\prime} 53^{\prime \prime}$ & $93^{\circ} 06^{\prime} 14^{\prime \prime}$ \\
\hline 09 & Huimanguillo & Chontalpa & $18^{\circ} 13^{\prime} 39^{\prime \prime}$ & $94^{\circ} 03^{\prime} 26^{\prime \prime}$ \\
\hline 10 & Cárdenas & Chontalpa & $18^{\circ} 12^{\prime} 37^{\prime \prime}$ & $94^{\circ} 00^{\prime} 23^{\prime \prime}$ \\
\hline 11 & Teapa & Sierra & $17^{\circ} 28^{\prime} 44^{\prime \prime}$ & $92^{\circ} 52^{\prime} 06^{\prime \prime}$ \\
\hline 12 & Teapa & Sierra & $17^{\circ} 28^{\prime} 48^{\prime \prime}$ & $92^{\circ} 52^{\prime} 06^{\prime \prime}$ \\
\hline 13 & Cunduacán & Chontalpa & $18^{\mathrm{o}} 17^{\prime} 44^{\prime \prime}$ & $93^{\circ} 12^{\prime} 48^{\prime \prime}$ \\
\hline 14 & Jalapa & Sierra & $17^{\circ} 44^{\prime} 26^{\prime \prime}$ & $92^{\circ} 42^{\prime} 41^{\prime \prime}$ \\
\hline 15 & Jalapa & Sierra & $17^{\circ} 44^{\prime} 26^{\prime \prime}$ & $92^{\circ} 42^{\prime} 33^{\prime \prime}$ \\
\hline 16 & Jalapa & Sierra & $17^{\circ} 41^{\prime} 27^{\prime \prime}$ & $92^{\circ} 42^{\prime} 08^{\prime \prime}$ \\
\hline 17 & Jalapa & Sierra & $17^{\circ} 45^{\prime} 39^{\prime \prime}$ & $92^{\circ} 51^{\prime} 15^{\prime \prime}$ \\
\hline 18 & Jalapa & Sierra & $17^{\mathrm{o}} 47^{\prime} 22^{\prime \prime}$ & $92^{\circ} 48^{\prime} 25^{\prime \prime}$ \\
\hline 19 & Jalapa & Sierra & $17^{\circ} 46^{\prime} 06^{\prime \prime}$ & $92^{\circ} 46^{\prime} 13^{\prime \prime}$ \\
\hline 20 & Teapa & Sierra & $17^{\circ} 34^{\prime} 00^{\prime \prime}$ & $92^{\circ} 52^{\prime} 31^{\prime \prime}$ \\
\hline 21 & Teapa & Sierra & $17^{\circ} 33^{\prime} 30^{\prime \prime}$ & $92^{\circ} 57^{\prime} 30^{\prime \prime}$ \\
\hline 22 & Nacajuca & Centro & $18^{\circ} 11^{\prime} 53^{\prime \prime}$ & $93^{\circ} 06^{\prime} 14^{\prime \prime}$ \\
\hline 23 & Nacajuca & Centro & $18^{\circ} 11^{\prime} 53^{\prime \prime}$ & $93^{\circ} 06^{\prime} 12^{\prime \prime}$ \\
\hline 24 & Nacajuca & Centro & $18^{\circ} 11^{\prime} 53^{\prime \prime}$ & $93^{\circ} 06^{\prime} 30^{\prime \prime}$ \\
\hline 25 & Jalpa de Méndez & Centro & $18^{\mathrm{o}} 00^{\prime} 23^{\prime \prime}$ & $92^{\circ} 52^{\prime} 40^{\prime \prime}$ \\
\hline 26 & Jalpa de Méndez & Centro & $18^{\circ} 11^{\prime} 53^{\prime \prime}$ & $93^{\circ} 06^{\prime} 14^{\prime \prime}$ \\
\hline 27 & Teapa & Sierra & $18^{\circ} 28^{\prime} 44^{\prime \prime}$ & $92^{\circ} 52^{\prime} 06^{\prime \prime}$ \\
\hline 28 & Tacotalpa & Sierra & $17^{\circ} 24^{\prime} 56^{\prime \prime}$ & $92^{\circ} 50^{\prime} 35^{\prime \prime}$ \\
\hline 29 & Tacotalpa & Sierra & $17^{\circ} 29^{\prime} 48^{\prime \prime}$ & $92^{\circ}-48^{\prime} 04^{\prime \prime}$ \\
\hline 30 & Comalcalco & Chontalpa & $18^{\circ} 13^{\prime} 38^{\prime \prime}$ & $93^{\circ} 39^{\prime} 52^{\prime \prime}$ \\
\hline 31 & Comalcalco & Chontalpa & $18^{\circ} 14^{\prime} 26^{\prime \prime}$ & $93^{\circ} 21^{\prime} 05^{\prime \prime}$ \\
\hline 32 & Comalcalco & Chontalpa & $18^{\circ} 13^{\prime} 33^{\prime \prime}$ & $93^{\circ} 18^{\prime} 22^{\prime \prime}$ \\
\hline 33 & Cárdenas & Chontalpa & $18^{\circ} 14^{\prime} 11^{\prime \prime}$ & $93^{\circ} 20^{\prime} 11^{\prime \prime}$ \\
\hline 34 & Tacotalpa & Sierra & $17^{\circ} 29^{\prime} 40^{\prime \prime}$ & $92^{\circ} 48^{\prime} 06^{\prime \prime}$ \\
\hline 35 & Tacotalpa & Sierra & $17^{\circ} 21^{\prime} 29^{\prime \prime}$ & $92^{\circ} 43^{\prime} 43^{\prime \prime}$ \\
\hline
\end{tabular}

del grano de cada población.

El trabajo se dividió en dos fases, en la primera se realizó la técnica de textura de disección de acuerdo con Leyva et al. (2002), para determinar el peso del pericarpio, germen, endospermo harinoso y endospermo vítreo. Se hicieron cinco repeticiones de 20 granos de cada población, que no presentaran daño por plagas o enfermedades. En esta fase se determinó el peso de 100 granos en una balanza analítica digital $(\mathrm{A} \& \mathrm{D}$, modelo
GR200, San José, California, USA).

En la segunda fase se evaluó la calidad fisiológica de los granos mediante la prueba de germinación estándar, para lo cual se seleccionó una muestra al azar de 100 semillas por población para establecer cuatro repeticiones de 25 semillas (Ramírez-Huerta et al., 2007). Previo a la prueba de germinación y vigor los granos se trataron con Vitavax $200 \mathrm{~F}$ (carboxin 17\% + thiram 17\%), para prevenir el crecimiento de hongos durante 
la germinación. Para la prueba de germinación estándar se utilizó el método entre papel, para ello se pusieron las semillas en toallas de papel anchor (Paper Company, St. Paul, Minnesota, USA) previamente humedecidas, para luego enrrollarlas para formar tacos, depositar en bolsas de plástico y colocarlas en un recipiente que se introdujo en una cámara germinadora (Thermo Scientific, Modelo $818 \AA$, Waltham, Massachusetts, USA) a $25 \pm 1^{\circ} \mathrm{C}$ por 7 días (Moreno 1996). Se evaluó el porcentaje de germinación, por medio del número de plántulas normales entre el número de semillas totales; plántulas anormales, las que presentaron malformaciones en raíz y plúmula, y semillas muertas, que se contó por medio de las semillas que no presentaron estructuras esenciales al finalizar la prueba (Moreno 1996). Para la prueba de vigor se seleccionaron al azar 100 semillas por población para realizar cuatro repeticiones de 25 semillas, para la prueba se utilizaron toallas de papel anchor de $30 \times 35 \mathrm{~cm}$, en las que se marco una línea en el eje de $30 \mathrm{~cm}$, para luego marcar cinco líneas paralelas a la línea central a distancia de $2 \mathrm{~cm}$ entre líneas, que se marcaron en la parte superior de la toalla de papel. Sobre la línea central se marcaron 25 puntos a $1 \mathrm{~cm}$ de distancia y se pegó un grano en cada punto, con el embrión de los granos hacia el lado contrario de las líneas y la plúmula apuntando hacia las líneas. Para luego poner encima de las semillas otra toalla de papel anchor, humedecer, enrrollar en sentido perpendicular a las líneas horizontales, colocar en bolsas de plástico transparente y poner en cámara germinadora a $25 \pm 1^{\circ} \mathrm{C}$ por 7 días con alta humedad relativa. Al finalizar los días de la prueba se determinó la longitud de plántula de las plántulas normales para medir el vigor, considerando: longitud menor de $3 \mathrm{~cm}$ como sin vigor; de 3 a $4,9 \mathrm{~cm}$ con bajo vigor, de 5 a 6,9 cm con mediano vigor, y mayor de $7 \mathrm{~cm}$ como vigor alto (Ruíz-Torres et al., 2012).

En ambas fases se utilizó un diseño completamente al azar, con cinco repeticiones en la primera y cuatro repeticiones en la segunda fase. Los resultados se sometieron a pruebas de homogeneidad y normalidad, en las variables que fue necesario transformar los datos se utilizó la $\sqrt{(x+0,5)}$. Las medias se compararon con la prueba de Tukey ( $\mathrm{p} \leq 0,05)$. Para conocer la relación entre las variables estructurales y morfológicas con los valores medios de las variables evaluadas se realizó un análisis de correlación canónica con el programa SAS 9.2 (SAS, 2003).

\section{RESULTADOS}

En las variables morfológicas y fisiológicas (Tabla 2), hubo diferencias estadísticas significativas $(p \leq 0,01)$ entre las 35 poblaciones de maíces criollos de la región Grijalva del estado de Tabasco, México. Mientras que la comparación de medias de Tukey $(\mathrm{p} \leq 0,05)$ detectó diferencias entre las variables estructurales (Tablas 3) y fisiológicas (Tabla 4) evaluadas.

El peso de grano presentó variaciones entre 26,06 y 37,44 g por cada 100 granos, con mayores pesos en las poblaciones $13(37,44 \mathrm{~g}), 16(37,22 \mathrm{~g})$, $27(36,30 \mathrm{~g}), 15(35,88 \mathrm{~g})$ y $33(35,82 \mathrm{~g})$, mientras que los menores pesos lo tuvieron las poblaciones 30 (26,06 g), 20 (26,34 g), 22 (26,40 g), 11 (26,58 g) y $31(26,62 \mathrm{~g})$. El peso de endospermo vítreo tuvo variaciones entre 0,068 y $0,209 \mathrm{~g}$, y el peso del endospermo harinoso fue de 0,067 a 0,152 $\mathrm{g}$; los mayores pesos de endospermo vítreo lo presentaron las poblaciones $34(0,210 \mathrm{~g}), 01(0,194$ g) y $33(0,172 \mathrm{~g})$; correspondiendo los mayores pesos de endospermo harinoso a las poblaciones 13 y 16 con 0,152 g. Para el contenido de pericarpio los valores oscilaron entre 0,016 (población 23) y $0,031 \mathrm{~g}$ (población 22), en tanto que para contenido de germen los valores oscilaron entre 0,019 (población 03) y 0,046 g (población 21).

En la Tabla 4 se observa que el $71 \%$ (25 poblaciones) presentaron una geminación mayor del $80 \%$, de las cuales las poblaciones 29,30 y 31 germinaron al $100 \%$, en tanto que el resto de las poblaciones tuvieron germinaciones entre el 28,00 y $74,67 \%$. El $60 \%$ (21) de las poblaciones tuvieron valores de longitud de plántula (vigor) mayores de $5 \mathrm{~cm}$, presentando la población 30 la mayor longitud $(9,27 \mathrm{~cm})$. Para la variable plantas normales el porcentaje fue del 16,00 al 97,33\%; hubo 18 poblaciones (51,43\%) mayores del $80 \%$; mientras que las poblaciones $5,16 \mathrm{y}$ $18(8,60 \%)$ tuvieron los menores porcentajes de plantas normales. La variable plantas anormales presentó porcentajes de 1,33 a $44 \%$, presentando la población 5 el mayor valor, y las poblaciones 20 , 22 y 25, los menores valores. Las semillas muertas oscilaron entre 1,33 a $61,33 \%$, el mayor valor lo tuvo la población $16(61,33 \%)$, mientras que los valores más bajos lo tuvieron las poblaciones 17 , 20, 22, 28, 31 y 35, con sólo el 1,33\% de semillas muertas.

La correlación canónica es una técnica multivariada que consiste en la construcción de pares sucesivos de variables canónicas, en combinaciones lineales de las variables originales, pudiendo conceptualizarse como entidades globalizadoras de las variables originales del grupo del cual se derivan, por lo que la variable canónica "Estructural 1" se puede interpretar 


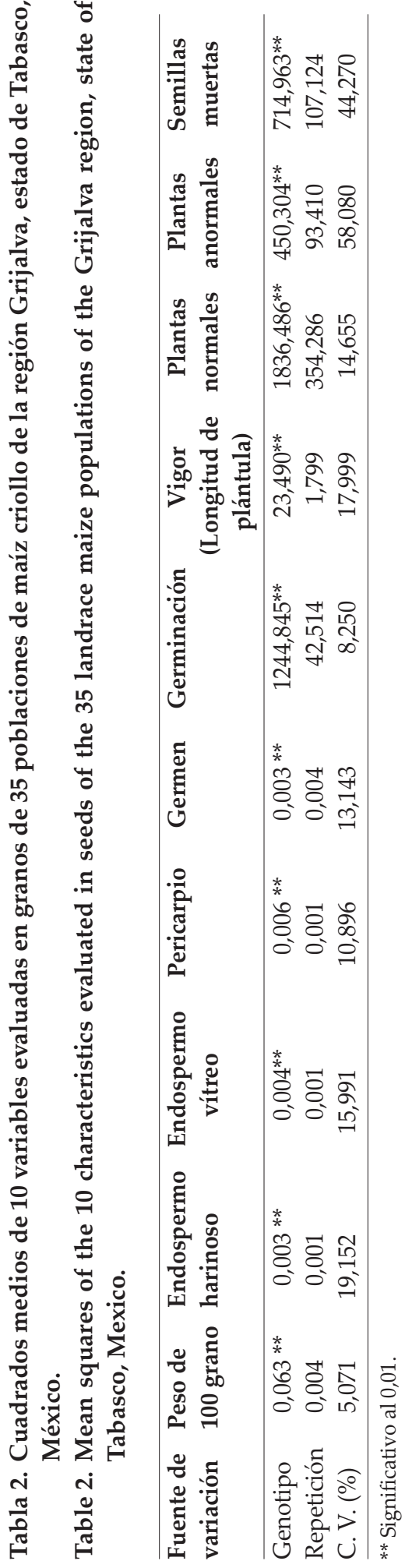

como una variable integradora de las diferentes proporciones que componen la semilla, mientras que la variable canónica "Fisiológica 1" representa una integración de los diferentes indicadores fisiológicos de vigor de la semilla. En la Fig. 1 se observa que la correlación entre la variable Estructural 1 y Fisiológica 1 fue significativa $(p=0,05)$ con un valor de 0,7051 . En la variable canónica Estructural 1 las variables originales con mayor influencia fueron el endospermo vítreo y peso de grano, mientras que la variable canónica Fisiológica 1, estuvo asociada de forma positiva con el número de plantas anormales, pero la asociación fue negativa con las variables vigor y germinación.

\section{DISCUSIÓN}

El análisis de varianza de todas las variables mostró diferencias estadísticas significativas $(p \leq 0,01)$ entre las poblaciones (Tabla 2), lo que se puede deber a la diversidad genética de las poblaciones evaluadas. Al respecto, Zhang et al. (2011) y González-Cortés et al. (2016), indicaron que las diferencias en las variables estructurales y fisiológicas de los granos de maíz se deben a la variabilidad genética de los mismos, debido a las proporciones de endospermo harinoso y vítreo que presentan los diferentes tipos de grano.

El peso de grano osciló entre 26,06 y 37,44 g, valores ligeramente superiores al rango de 27,20 a 33,70 g reportados por Salinas et al. (2013) para maíces tropicales (Tabla 3). En general, los granos de las 35 poblaciones evaluadas se pueden clasificar como de tamaño medio, en comparación con los granos de tamaño grande de las razas Ancho y Cacahuacintle (60 - 70 g por 100 granos), o pequeño en las razas Palomero Toluqueño o Arrocillo (18 - $22 \mathrm{~g}$ por 100 granos) (Salinas et al., 2012). La proporción relativa de endospermo vítreo y harinoso en un grano de maíz está asociada con la textura del endospermo, la cual influye en el potencial de almacenamiento y las características de procesamiento (González et al., 2014). Al respecto el 71,4\% (25) de las poblaciones evaluadas tuvieron una mayor proporción de endospermo vítreo, mientras que el 28,6\% (10) de las poblaciones tuvieron una mayor proporción de endospermo harinoso, lo que indica que la mayor proporción de poblaciones de maíces tienen granos con dureza intermedia a dura, lo que coincide con Salinas et al. (2012) quienes indicaron que la mayoría de los maíces tropicales tiene endospermo de intermedio a duro. Sobre la diversidad de la proporción de endospermo vítreo y harinoso, Pérez et al. (2007) indicaron que los factores genéticos son los que más afectan la proporción de endospermo en los granos de 
Tabla 3. Comparación de medias para las variables estructurales de granos de 35 poblaciones de maíz criollo de la región Grijalva, estado de Tabasco, México.

Table 3. Comparison of means for structural characteristics of seeds of the 35 landrace maize populations of the Grijalva region, state of Tabasco, Mexico.

\begin{tabular}{|c|c|c|c|c|c|}
\hline Población & $\begin{array}{l}\text { Peso de } 100 \\
\text { granos (g) }\end{array}$ & $\begin{array}{c}\text { Endospermo } \\
\text { harinoso } \\
\text { (g) }\end{array}$ & $\begin{array}{c}\text { Endospermo } \\
\text { vítreo } \\
\text { (g) }\end{array}$ & $\begin{array}{l}\text { Pericarpio } \\
\text { (g) }\end{array}$ & $\begin{array}{c}\text { Germen } \\
\text { (g) }\end{array}$ \\
\hline 01 & $31,620^{\text {bcdef }}$ & $0,078^{\text {efg }}$ & $0,194^{\mathrm{ab}}$ & $0,023^{\text {cdefghi }}$ & $0,021^{\mathrm{ij}}$ \\
\hline 02 & $30,000^{\text {efghi }}$ & $0,097^{\text {bcdefg }}$ & $0,152^{\text {bcde }}$ & $0,021^{\text {defghij }}$ & $0,029^{\text {defghi }}$ \\
\hline 03 & $27,000^{\text {hij }}$ & $0,068^{\mathrm{g}}$ & $0,160^{\mathrm{abcd}}$ & $0,023^{\text {cdefghi }}$ & $0,019^{j}$ \\
\hline 04 & $30,900^{\text {cdefg }}$ & $0,096^{\text {bcdefg }}$ & $0,170^{\mathrm{abc}}$ & 0,019ghij & $0,024^{\text {ghij }}$ \\
\hline 05 & $30,560^{\text {defgh }}$ & $0,098^{\text {bcdefg }}$ & $0,157^{\text {abcde }}$ & $0,022^{\text {defghi }}$ & $0,029^{\text {defghi }}$ \\
\hline 06 & $31,760^{\text {bcdef }}$ & $0,107^{\text {abcdefg }}$ & $0,155^{\text {bcde }}$ & $0,025^{\text {abcdefg }}$ & $0,030^{\text {defghi }}$ \\
\hline 07 & $27,660^{\text {ghij }}$ & $0,101^{\text {abcdefg }}$ & $0,130^{\text {cdefgh }}$ & $0,024^{\text {bcdefghi }}$ & $0,023^{\text {hij }}$ \\
\hline 08 & $28,020^{\text {fghij }}$ & $0,109^{\text {abcdefg }}$ & $0,131^{\text {cdefgh }}$ & $0,022 \mathrm{~d}^{\text {efghij }}$ & $0,028^{\text {defghij }}$ \\
\hline 09 & $34,740^{\mathrm{ab}}$ & $0,143^{\mathrm{abc}}$ & $0,147^{\text {bcdef }}$ & $0,023^{\text {cdefghi }}$ & $0,033^{\text {cdefg }}$ \\
\hline 10 & $28,080^{\text {fghij }}$ & $0,110^{\text {abcdefg }}$ & $0,128^{\text {cdefgh }}$ & 0,019ghij & $0,024^{\text {ghij }}$ \\
\hline 11 & $26,580^{\mathrm{ij}}$ & $0,098^{\text {bcdefg }}$ & $0,123^{\text {cdefgh }}$ & $0,018^{\mathrm{hij}}$ & $0,025^{\text {fghij }}$ \\
\hline 12 & $28,600^{\text {fghij }}$ & 0,109abcdefg & $0,131^{\text {cdefgh }}$ & $0,024^{\text {bcdefgh }}$ & $0,021^{\mathrm{ij}}$ \\
\hline 13 & $37,440^{\mathrm{a}}$ & $0,152^{\mathrm{a}}$ & $0,163^{\mathrm{abcd}}$ & $0,024^{\text {bcdefghi }}$ & $0,035^{\text {bcde }}$ \\
\hline 14 & $28,060^{\text {fghij }}$ & $0,104^{\text {abcdefg }}$ & $0,127^{\text {cdefgh }}$ & $0,021^{\text {efghij }}$ & $0,029^{\text {defghij }}$ \\
\hline 15 & $35,880^{a}$ & $0,128^{\text {abcde }}$ & $0,166^{\mathrm{abcd}}$ & $0,029 \mathrm{abc}$ & $0,036^{\mathrm{abcd}}$ \\
\hline 16 & $37,220^{\mathrm{a}}$ & $0,152^{\mathrm{a}}$ & $0,159^{\mathrm{abcd}}$ & $0,025^{\text {abcdefg }}$ & $0,037^{\mathrm{abcd}}$ \\
\hline 17 & $28,040^{\text {fghij }}$ & $0,091^{\text {cdefg }}$ & $0,134^{\text {cdefg }}$ & $0,024^{\text {bcdefgh }}$ & $0,030^{\text {defghi }}$ \\
\hline 18 & $27,140^{\text {ghij }}$ & $0,128^{\text {abcde }}$ & $0,098^{\mathrm{fgh}}$ & $0,023^{\text {cdefghi }}$ & $0,022^{\text {hij }}$ \\
\hline 19 & $34,240^{\mathrm{abcd}}$ & $0,145^{\mathrm{abc}}$ & $0,138^{\text {cdefg }}$ & $0,026^{\text {abcdef }}$ & $0,034^{\text {cdef }}$ \\
\hline 20 & $26,340^{\mathrm{ij}}$ & $0,133^{\mathrm{abcd}}$ & $0,085^{\mathrm{gh}}$ & $0,018^{\mathrm{ij}}$ & $0,028^{\text {defghij }}$ \\
\hline 21 & $33,760^{\text {abcde }}$ & $0,143^{\mathrm{abc}}$ & $0,124^{\text {cdefgh }}$ & $0,025^{\text {abcdefg }}$ & $0,046^{\mathrm{a}}$ \\
\hline 22 & $26,400^{\mathrm{ij}}$ & $0,110^{\text {abcdefg }}$ & $0,079^{\mathrm{h}}$ & $0,031^{\mathrm{a}}$ & $0,044^{\mathrm{ab}}$ \\
\hline 23 & $27,400^{\text {ghij }}$ & $0,132^{\mathrm{abcd}}$ & $0,105^{\text {efgh }}$ & $0,016^{j}$ & $0,022^{\text {hij }}$ \\
\hline 24 & $29,260^{\text {fghij }}$ & $0,123^{\text {abcdef }}$ & $0,112^{\text {defgh }}$ & $0,027^{\text {abcde }}$ & $0,032^{\text {defgh }}$ \\
\hline 25 & $30,920^{\text {cdefg }}$ & $0,132^{\text {abcde }}$ & $0,155^{\text {defgh }}$ & $0,027^{\text {abcde }}$ & $0,03^{5 \text { bcde }}$ \\
\hline 26 & $27,300^{\text {ghij }}$ & $0,130^{\text {abcde }}$ & $0,097^{\mathrm{fgh}}$ & $0,019^{\text {fghij }}$ & $0,026^{\text {efghij }}$ \\
\hline 27 & $36,300^{a}$ & $0,147^{\mathrm{ab}}$ & $0,146^{\text {bcdef }}$ & $0,028^{\mathrm{abcd}}$ & $0,042^{\mathrm{abc}}$ \\
\hline 28 & $29,580^{\text {fghij }}$ & $0,145^{\text {abcde }}$ & $0,120^{\text {cdefgh }}$ & $0,027^{\text {abcde }}$ & $0,024^{\text {ghij }}$ \\
\hline 29 & $26,780^{\mathrm{hij}}$ & $0,099^{\text {abcdefg }}$ & $0,121^{\text {cdefgh }}$ & $0,021^{\text {efghij }}$ & $0,027^{\text {defghij }}$ \\
\hline 30 & $26,060^{j}$ & 0,089 defg & $0,124^{\text {cdefgh }}$ & $0,023^{\text {cdefghi }}$ & $0,025^{\text {fghij }}$ \\
\hline 31 & $26,620^{\mathrm{ij}}$ & $0,101^{\text {abcdefg }}$ & $0,121^{\text {cdefgh }}$ & $0,022^{\text {defghij }}$ & $0,023^{\mathrm{hij}}$ \\
\hline 32 & $29,080^{\text {fghij }}$ & $0,099^{\text {abcdefg }}$ & $0,129^{\text {cdefgh }}$ & $0,026^{\text {abcde }}$ & $0,036^{\mathrm{abcd}}$ \\
\hline 33 & $35,820^{a}$ & $0,127^{\text {abcdefg }}$ & $0,172^{\mathrm{abc}}$ & $0,024^{\text {cdefghi }}$ & $0,043^{\mathrm{abc}}$ \\
\hline 34 & $34,500^{\mathrm{abc}}$ & $0,070^{\mathrm{fg}}$ & $0,210^{\mathrm{a}}$ & $0,030^{\mathrm{ab}}$ & $0,035^{\text {bcde }}$ \\
\hline 35 & $29,160^{\text {fghij }}$ & $0,116^{\text {abcdefg }}$ & $0,131^{\text {cdefgh }}$ & $0,022^{\text {defghij }}$ & $0,023^{\text {hij }}$ \\
\hline
\end{tabular}

Medias con la misma letra no son significativamente diferentes $(p \leq 0,05)$.

maíz, debido a que el genotipo tiene un papel importante en la composición de los componentes de la semilla.

El peso de pericarpio varió entre 0,016 y 0,030 $\mathrm{g}$, encontrándose poblaciones con porcentajes mayores al $5 \%$ de pericarpio, como la población 22 , lo que es importante, debido a que el pericarpio es la parte del grano que tiene elevado contenido de fibra cruda (Nuss y Tanumihardjo, 2010). Por otra parte pericarpios gruesos influyen en forma desfavorable en la fisiología de la semilla y en el establecimiento temprano del cultivo (Vicient, 2017), por un probable retraso en la imbibición por la interferencia física de la protusión de 
Tabla 4. Comparación de medias para las variables fisiológicas de granos de 35 poblaciones de maíz criollo de la región Grijalva del estado de Tabasco, México.

Table 4. Comparison of means for physiological characteristics in seeds of the 35 landrace maize populations of the Grijalva region of the state of Tabasco, Mexico.

\begin{tabular}{|c|c|c|c|c|c|}
\hline Población & $\begin{array}{c}\text { Germinación } \\
(\%)\end{array}$ & Vigor (cm) & $\begin{array}{c}\text { Plantas } \\
\text { normales (\%) }\end{array}$ & $\begin{array}{l}\text { Plantas } \\
\text { anormales (\%) }\end{array}$ & $\begin{array}{l}\text { Semillas } \\
\text { muertas (\%) }\end{array}$ \\
\hline 01 & $52,00^{\mathrm{gh}}$ & $2,05^{\text {ghij }}$ & $40,00^{\text {defg }}$ & $30,67^{\text {abcde }}$ & $29,33^{\text {bcde }}$ \\
\hline 02 & $61,33^{\text {efgh }}$ & $3,48^{\text {fghij }}$ & $68,00^{\text {abcde }}$ & $6,67^{\text {def }}$ & $25,33^{\text {bcdefg }}$ \\
\hline 03 & $94,67^{\mathrm{ab}}$ & $7,84^{\mathrm{abcd}}$ & $90,67^{a}$ & $4,00^{\mathrm{ef}}$ & $5,33^{\mathrm{gh}}$ \\
\hline 04 & $29,33^{\mathrm{i}}$ & $1,60^{\mathrm{ij}}$ & $37,33^{\text {efg }}$ & $25,33^{\text {abcdef }}$ & $37,33^{\mathrm{bcd}}$ \\
\hline 05 & $68,00^{\text {cdefg }}$ & $0,72^{j}$ & $16,00^{\mathrm{g}}$ & $44,00^{\mathrm{a}}$ & $40,00^{\mathrm{bc}}$ \\
\hline 06 & $81,33^{\text {abcde }}$ & $2,29^{\text {ghij }}$ & $45,33^{\text {cdefg }}$ & $33,33^{\mathrm{abcd}}$ & $21,33^{\text {cdefgh }}$ \\
\hline 07 & $64,00^{\text {defgh }}$ & $2,12^{\text {ghij }}$ & $36,00^{\text {efg }}$ & $21,33^{\text {abcdef }}$ & $42,67^{\mathrm{ab}}$ \\
\hline 08 & $88,00^{\mathrm{abc}}$ & $4,45^{\text {efghi }}$ & $72,00^{\mathrm{abcd}}$ & $18,67^{\text {abcdef }}$ & $9,33^{\text {efgh }}$ \\
\hline 09 & $45,33^{\mathrm{hi}}$ & $1,92^{\mathrm{hij}}$ & $45,33^{\text {cdefg }}$ & $29,33^{\text {abcdef }}$ & $25,33^{\text {bcdefg }}$ \\
\hline 10 & $97,33^{a}$ & $4,96^{\text {defgh }}$ & $72,00^{\mathrm{abcd}}$ & $22,67^{\text {abcdef }}$ & $5,33^{\mathrm{gh}}$ \\
\hline 11 & $92,00^{\mathrm{ab}}$ & $6,53^{\text {abcdef }}$ & $82,67^{\mathrm{a}}$ & $5,33^{\text {def }}$ & $12,00^{\text {efgh }}$ \\
\hline 12 & $89,33^{\mathrm{abc}}$ & $5,21^{\text {cdefg }}$ & $65,33^{\text {abcdef }}$ & $21,33^{\text {abcdef }}$ & $13,33^{\text {efgh }}$ \\
\hline 13 & $85,33^{\mathrm{abcd}}$ & $2,15^{\text {ghij }}$ & $46,67^{\text {bcdefg }}$ & $33,33^{\mathrm{abcd}}$ & $20,00^{\text {cdefgh }}$ \\
\hline 14 & $74,67^{\text {bcdef }}$ & $2,20^{\text {ghij }}$ & $33,33^{\mathrm{fg}}$ & $40,00^{\mathrm{ab}}$ & $26,67^{\text {bcdef }}$ \\
\hline 15 & $68,00^{\text {cdefg }}$ & $3,83^{\text {fghij }}$ & $75,33^{\mathrm{abcd}}$ & $9,33^{\text {def }}$ & $17,33^{\text {defgh }}$ \\
\hline 16 & $28,00^{\mathrm{i}}$ & $2,01^{\text {ghij }}$ & $30,67^{g}$ & $8,00^{\text {def }}$ & $61,33^{\mathrm{a}}$ \\
\hline 17 & $98,67^{\mathrm{a}}$ & $9,19^{a}$ & $94,67^{a}$ & $4,00^{\mathrm{ef}}$ & $1,33^{\mathrm{h}}$ \\
\hline 18 & $56,00^{\mathrm{fgh}}$ & $0,97^{i}$ & $17,33^{\mathrm{g}}$ & $38,67^{a b c}$ & $44,00^{\mathrm{ab}}$ \\
\hline 19 & $94,67^{\mathrm{ab}}$ & $7,92^{\mathrm{abcd}}$ & $88,00^{a}$ & $8,00^{\text {def }}$ & $4,00^{\mathrm{h}}$ \\
\hline 20 & $98,67^{a}$ & $8,76^{\mathrm{ab}}$ & $97,33^{a}$ & $1,33^{\mathrm{f}}$ & $1,33^{\mathrm{h}}$ \\
\hline 21 & $98,67^{a}$ & $6,33^{\text {abcdef }}$ & $94,67^{a}$ & $2,67^{\text {ef }}$ & $2,67^{\mathrm{h}}$ \\
\hline 22 & $96,00^{\mathrm{ab}}$ & $8,33^{\mathrm{abc}}$ & $97.33^{\mathrm{a}}$ & $1,33^{\mathrm{f}}$ & $1,33^{\mathrm{h}}$ \\
\hline 23 & $94,67^{\mathrm{ab}}$ & $9,15^{\mathrm{a}}$ & $88,00^{\mathrm{a}}$ & $4,00^{\mathrm{ef}}$ & $8,00^{\mathrm{fgh}}$ \\
\hline 24 & $97,33^{a}$ & $6,45^{\text {abcdef }}$ & $88,00^{\mathrm{a}}$ & $9,33^{\text {def }}$ & $2,67^{\mathrm{h}}$ \\
\hline 25 & $97,33^{\mathrm{a}}$ & $8,03^{\mathrm{abcd}}$ & $96,00^{a}$ & $1,33^{\mathrm{f}}$ & $2,67^{\mathrm{h}}$ \\
\hline 26 & $86,67^{\mathrm{abc}}$ & $5,72^{\text {bcdef }}$ & $81,33^{a}$ & $9,33^{\text {def }}$ & $9,33^{\text {efgh }}$ \\
\hline 27 & $92,00^{\mathrm{ab}}$ & $5,62^{\text {bcdef }}$ & $80,00^{\mathrm{ab}}$ & $3,00^{\mathrm{abcd}}$ & $8,00^{\text {fgh }}$ \\
\hline 28 & $97,33^{a}$ & $7,67^{\text {abcde }}$ & $89,33^{a}$ & $9,33^{\text {def }}$ & $1,33^{\mathrm{h}}$ \\
\hline 29 & $100,00^{\mathrm{a}}$ & $8,61^{\mathrm{ab}}$ & $85,33^{a}$ & $10,67^{\text {cdef }}$ & $4,00^{\mathrm{h}}$ \\
\hline 30 & $100,00^{\mathrm{a}}$ & $9,27^{a}$ & $92,00^{\mathrm{a}}$ & $5,33^{\mathrm{def}}$ & $2,67^{\mathrm{h}}$ \\
\hline 31 & $100,00^{a}$ & $8,60^{\mathrm{ab}}$ & $86,67^{a}$ & $12,00^{\text {bcdef }}$ & $1,33^{\mathrm{h}}$ \\
\hline 32 & $96,00^{\mathrm{ab}}$ & $7,83^{\mathrm{abcd}}$ & $84,00^{\mathrm{a}}$ & $12,00^{\text {bcdef }}$ & $4,00^{\mathrm{h}}$ \\
\hline 33 & $98,67^{a}$ & $7,96^{\mathrm{abcd}}$ & $86,67^{\mathrm{a}}$ & $10,67^{\text {cdef }}$ & $2,67^{\mathrm{h}}$ \\
\hline 34 & $82,67^{\text {abcde }}$ & $6,11^{\text {abcdef }}$ & $77,33^{\mathrm{abc}}$ & $6,67^{\mathrm{def}}$ & $16,00^{\text {efgh }}$ \\
\hline 35 & $92,00^{\mathrm{ab}}$ & $7,12^{\text {abcde }}$ & $85,33^{a}$ & $13,33^{\text {bcdef }}$ & $1,33^{\mathrm{h}}$ \\
\hline
\end{tabular}

Medias con la misma letra no son significativamente diferentes $(p \leq 0,05)$.

las estructuras iniciales como la radícula y el coleóptilo (Pérez et al., 2007). En lo referente al peso de germen, la población 21 tuvo el mayor valor con $0,046 \mathrm{~g}$ y la población 03 el menor valor con $0,019 \mathrm{~g}$; de las 35 poblaciones estudiadas, el $40 \%$ (14) tuvieron valores mayores del $10 \%$ de germen; al respecto esta parte del grano es importante porque contiene hasta un $83 \%$ del total de los lípidos, el 70\% del azúcar y el 26\% de la proteína del grano (Koehler y Wieser, 2013).

La prueba de germinación estándar detectó diferencias estadísticas significativas entre las poblaciones (Tabla 4), con porcentajes de germinación entre 28,00 y $100 \%$. De las 35 poblaciones evaluadas el $71,4 \%$ (25) tuvieron porcentajes de germinación mayores del $80 \%$. 


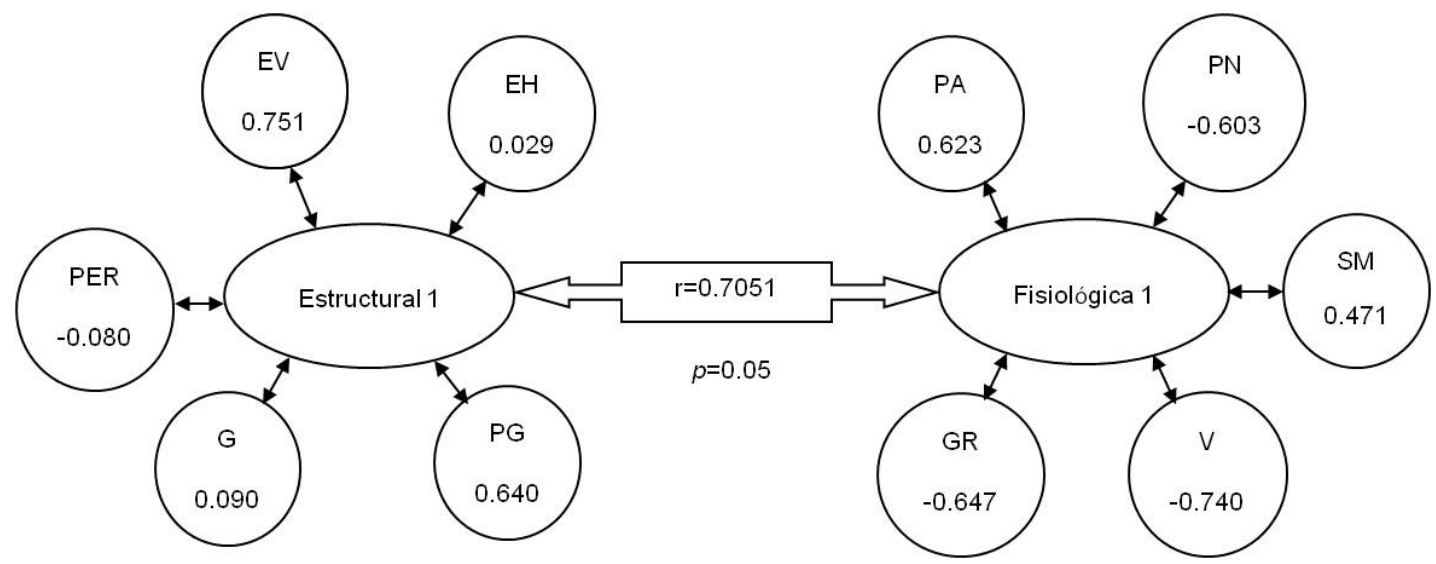

Fig. 1. Fracción de la estructura canónica mostrando la primera variable canónica de cada grupo y la correlación con sus respectivas variables originales. Estructural 1 = Variables estructurales; $\mathrm{EH}=$ Endospermo harinoso; EV = Endospermo vítreo; $\mathrm{PER}=$ Pericarpio; $\mathrm{G}=$ Germen; $\mathrm{PG}=$ Peso de grano; Variable fisiológica 1 = Variables fisiológicas; PA = Plantas anormales; PN = Plantas normales; $\mathrm{SM}=$ Semillas muertas; $\mathrm{V}=$ longitud de plúmula; y GR = Germinación.

Fig. 1. Canonical structure of the first canonical variable for each group and its correlation with the original variables. Structural 1 = Structural variable; $\mathrm{EH}=$ Floury endosperm; EV = Vitreous endosperm; PER = Pericarp; GE = Germ; PG = Grain weight; Physiological variable $1=$ Physiological characteristics; PA = Abnormal plants; PN = Normal plants; SM = Dead seeds; V = Vigor; and GR = Germination .

Valores similares de germinación en maíces criollos fueron reportados por GonzálezCortés et al. (2016). Al respecto, Aristizábal y Álvarez (2006) señalan que las semillas con germinación superior a $80 \%$ pueden clasificarse como de alta germinación, entre 60 y $80 \%$ como germinación media, y menores de $60 \%$ como baja germinación. Con base en esto, el 71,4\% de las poblaciones tendrían alta germinación, el $14,3 \%$ (5) germinación media, y el 14,3\% (5) baja germinación. En lo referente al vigor (longitud de plántula) se encontraron plántulas con tamaños entre 0,72 y $9,27 \mathrm{~cm}$, en general las poblaciones con mayor vigor tuvieron mayor proporción de endospermo vítreo; al respecto Pérez et al. (2007) encontraron que los maíces con endospermo vítreo tienen plántulas con mayor vigor, germinación y plántulas normales, lo que indica que el endospermo vítreo es más vigoroso que el endospermo harinoso. Se encontró que el $60 \%$ (21) de las poblaciones tienen longitudes de plántula mayores de $5 \mathrm{~cm}$, y el $40 \%$ (14) de las poblaciones tuvieron plántulas con alto vigor. Las poblaciones 17 y 30 tuvieron los mayores porcentajes de germinación y vigor, lo que coincide con Marcos-Filho (2015), quien indicó que los factores que influyen en el potencial fisiológico de la semilla son la germinación y el vigor. Las plántulas que se obtienen durante una prueba de germinación se clasifican como normales o anormales; las normales poseen estructuras adecuadas de raíces y brotes esenciales para su desarrollo posterior, y las anormales no tienen capacidad para desarrollarse y sufren deficiencia, descomposición o debilidad en sus sistemas de raíces y brotes (AOSA, 2014). $\mathrm{Al}$ respecto se observó que la población 05 tuvo el mayor porcentaje de plantas anormales y el menor porcentaje de plantas normales, además un bajo vigor con alto porcentaje de semillas muertas, lo que indica que ambas variables están relacionadas.

La correlación canónica (Fig. 1) muestra que para la variable integradora Estructural 1, las variables originales con mayor influencia fueron el peso de endospermo vítreo y el peso de grano. Mientras que la variable canónica Fisiológica 1 estuvo asociada con valores positivos en las variables originales de número de plantas anormales y semillas muertas, mientras que las variables vigor, germinación y plantas normales tuvieron influencia negativa. Estos resultados concuerdan con los de Pérez et al. (2007) quienes reportaron correlaciones canónicas similares para variables estructurales y fisiológicas de semillas de maíz. De acuerdo al valor de correlación $(r=0,7051)$ y la significancia $(p=0,05)$, los componentes de determinación estructural están relacionados de forma directa con la calidad fisiológica de la semilla de maíz. 


\section{CONCLUSIONES}

Se observó en general que las poblaciones que tuvieron alto vigor y germinación provienen de poblaciones que tienen granos con mayor proporción de endospermo vítreo. La relación entre las variables canónicas Estructural 1 y Fisiológica 1 indica que la variable endospermo vítreo se relaciona con las variables germinación y vigor.

\section{LITERATURA CITADA}

AOSA. 2014. Rules for testing seeds. Association of Official Seed Analysts (AOSA), USA. Available at http://www.aosaseed.com/aosa rules_for_testing_seeds (Accessed $10 \mathrm{Feb}$. 2016).

Aristizábal, L.M., y L.P. Álvarez. 2006. Los efectos del nivel de vigor de la semilla pueden persistir e influenciar el crecimiento de la planta, la uniformidad de la plantación y la productividad. Agronomía 14(1):17-24.

González-Cortés, N., H. Silos-Espino, J.C. Estrada Cabral, J.A. Chávez-Muñoz, y L. Tejero Jiménez. 2016. Características y propiedades del maíz (Zea mays L.) criollo cultivado en Aguascalientes, México. Rev. Mex. Cienc. Agríc. 6(3):669-680.

González, R.F., D. León, L. Borges, L. Pinzón, M. Magaña, R. Sanguines, y M. Urrestarazu. 2014. Envejecimiento acelerado sobre la calidad de semilla de maíz para producir germinados para forraje alternativo. Rev. Mex. Cienc. Agríc. (Pub. Esp. 8):1487-1493.

INEGI. 2011. Perspectiva estadística Tabasco. Instituto Nacional de Estadística y Geografía, Aguascalientes, México.

Kato, Y.T.A., C. Mapes, L.M. Mera, J.A. Serratos, y R.A. Bye. 2009. Origen y diversificación del maíz: una revisión analítica. Universidad Nacional Autónoma de México. Comisión Nacional para el Conocimiento y Uso de la Biodiversidad, Distrito Federal, México.

Koehler, P., and H. Wieser. 2013. Chemistry of cereal grains. p. 11-45. In Gobbetti, M., Gänzle, M. (eds.). Handbook on sourdough biotechnology. Springer U.S. New Delhi, India.

Leyva, O.O.R., A. Carballo, J.A. Mejía, y M.G. Vázquez. 2002. Procesamiento de imágenes para la estimación de textura de endospermo en líneas de maíz. Rev. Fitotec. Mex. 25(4):355-365.

Marcos-Filho, J. 2015. Seed vigor testing: an overview of the past, present and future perspective. Sci. Agric. 72(4):363-374.
Mariaca, M,R., E.J. Cano, y M. Sánchez. 2014. La milpa en la región Sierra ChiapasTabasco de Huitiapan, Tacotalpa. p. 329359. En González-Espinosa, M., y M.C. Brunel (Comp.) Montañas pueblos y agua: Dimensiones y realidades de la cuenca Grijalva. El Colegio de la Frontera Sur, Distrito Federal, México.

Moreno, M.E. 1996. Análisis físico y biológico de semillas agrícolas. 3a . ed. Programa Universitario de Alimentos. FAO, UNAM, México.

Navarro, M., G. Febles, and R.S. Herrera. 2015. Vigor: essential element for seed quality. Cuban J. Agric. Sci. 49 (4):447-458.

Nuss, E.T., and S.A. Tanumihardjo. 2010. Maize: A paramount staple crop in the context of global nutrition. Compr. Rev. Food Sci. Food Saf. 9:417-436.

Pérez, de la C.F.J., A. Carballo, A. Santacruz, A Hernández, y J.C. Molina. 2007. Calidad fisiológica en semillas de maíz con diferencias estructurales. Agric. Téc. Méx. 33(1):53-61

Pérez, M.C., A. Hernández, V.V. González, G. García, A. Carballo, T.R. Vásquez, y M.R. Tovar. 2006. Tamaño de semilla y relación con su calidad fisiológica en variedades de maíz para forraje. Agric. Téc. Méx. 32(3):341352.

Ramírez-Huerta, M.E., R. Ramírez-Zamora, J. Rivera-Camacho, y P.A. Romero-Huerta. 2007. Laboratorio de semillas del Campo Experimental Centro Altos de Jalisco. Instituto Nacional de Investigaciones Forestales, Agrícolas y Pecuarias. Publicación Especial Núm. 2, Jalisco, México.

Rosental, L., H. Nonogaki, and A. Fait. 2014. Activation and regulation of primary metabolism during seed germination. Seed Science Research 24(1):1-15.

Ruíz-Álvarez, O., R. Ortega-Ramírez, M.A. Vázquez-Peña, R.E. Ontiveros, y R. LópezLópez. 2012. Balance hídrico y clasificación climática del estado de Tabasco, México. Universidad y Ciencia 28(1):1-14.

Ruíz-Torres, N.A., F. Rincón-Sánchez, V.M. Bautista-Morales, J. M. Martínez-Reyna, H. C. Burciaga-Dávila, y M. Olvera-Esquivel. 2012. Calidad fisiológica de semilla en dos poblaciones de maíz criollo mejorado. Agraria 9(2):43-48.

Salinas, M.Y., F. Aragón, C. Ybarra, J. Aguilar, B. Altunar, y E. Sosa. 2013. Caracterización física y composición química de razas de maíz de grano azul/morado de las regiones tropicales y subtropicales de Oaxaca. Rev. Fitotec. Mex. 36(1): 23-31. 
Salinas, M.Y., F.J. Cruz, S.A. Díaz, y F. Castillo. 2012. Granos de maíces pigmentados de Chiapas, características físicas, contenido de antocianinas y valor nutracéutico. Rev. Fitotec. Mex. 35(1): 33.41

SAS. 2003. Statistical Analysis System. The analyst application. 2nd. ed. SAS Institute Inc., Cary, North Carolina, USA.

Vázquez, C.M.G., J.P. Pérez , J.M. Hernández , M.L. Marrufo, y E. Martínez. 2010. Calidad de grano y de tortillas de maíces criollos del altiplano y Valle del Mezquital, México. Rev. Fitotec. Mex. 33(4):49-56.
Vicient, C.M. 2017. The efect of frequency-specifc sound signals on the germination of maize seeds. BMC Res. Notes 10:323. doi:10.1186/ s13104-017-2643-4.

Vitoria, H., y J.R. Méndez. 2007. Relación de la calidad fisiológica de semillas de maíz con $\mathrm{pH}$ y conductividad eléctrica. Rev. Fac. Agrar. Univ. Nac. Cuyo 39(2):91-100.

Zhang, H., R. Goa, and S. Dong. 2011. Anatomical and physiological characteristics associated with corn endosperm texture. Agronomy Journal 103(4):1258-1264. 